\title{
On a method for determination of Poisson's ratio and Young modulus of a material
}

\author{
Vladimir B. Zelentsov ${ }^{1}$, Evgeniy V. Sadyrin ${ }^{1, *}$, Aleksandr G. Sukiyazov ${ }^{1}$, Nataliya Yu. \\ Shubchinskaya ${ }^{1,2}$ \\ ${ }^{1}$ Research and Education Center "Materials", Don State Technical University, 344000 \\ Rostov-on-Don, Russia \\ ${ }^{2}$ Mathematical Modeling Department, Southern Federal University, 344006 Rostov-on-Don, Russia
}

\begin{abstract}
On the base of modernized NanoTest 600 Platform 3 indentation method is proposed to determine elastic parameters - Poisson's ratio and Young's modulus - of a material while loading in an elastic region. The experiment is based on procedure: lateral surface of indenter tip with the shape of parabolic cylinder penetrates into the specimen. NanoTest 600 was equipped by additional optics, backlight and device for spatial orientation of the specimen. This modernization allows to control the process of the indenter penetration both along its length and from the edges, so that one can observe and measure the width of the contact area and control the depth of the indentation area in a sample material. Mathematical modeling of the indentation process was conducted within the framework of plane theory of elasticity. This required solution of the contact problem on indentation of a rigid indenter with a parabolic shape into an elastic strip coupled with a non-deformable substrate. The fulfilment of condition of zeroing the contact stresses at the edges of the indenter with a known width of the contact area allows to determine the Poisson's ratio, and condition of static equilibrium of the contact problem helps to find Young's modulus of a strip material.
\end{abstract}

\section{Introduction}

At present, the practice of determining the Young's modulus by means of indentation is widely applied. Nowadays, there are a sufficient number of methods for determining the Young's modulus. The most commonly use techniques in determining the Young's modulus were designed by Oliver and Pharr [1], Field and Swain [2]. Modern electronics and optics allow to increase significantly the level of measurement of physical indentation parameters and the automation of the indentation process at the hardware level, to improve as well the level of visualization of the indentation process both at the micro- and nanoscale and to improve software to support the indentation process. The increasing of level of mathematical description of the indentation process and its technical level allows us to bring maximally together the functional of the indentation model with the functionality of the experimental setup, which raises the efficiency of the method for characterization of

\footnotetext{
* Corresponding author: rainesquall@icloud.com
} 
material elastic parameters. Upgrading NanoTest 600 Platform 3 in order to increase functionality was carried out as follow: improving the shape of the indenter, sample orientation devices, empowering visualization of the process of indentation by using a video camera and optics, improving of mathematical modeling of the indentation process and other. All this made it possible to develop a sufficiently effective method for determining the Poisson's ratio and the Young's modulus of the sample material when it is indented in the elastic loading region.

\section{Special indentation procedure}

A special indentation procedure is understood as the process of pressing indenter of a special form into a sample material. The special form of the indenter is represented by a rectangular plate, whose edge is sharpened in the form of a parabolic cylinder (Fig. 1). The indenter 1 of the parabolic shape is pressed by force $P$ towards the material of sample 2 (Fig. 2), in such a way that the width of the contact area $2 a$ is visible from the edge of the indenter, either one can see the penetration depth of the indenter $\varepsilon$ into specimen (Fig. 3). It should be noted that the transverse size of the sample coincides with the length of the indenter $d$.

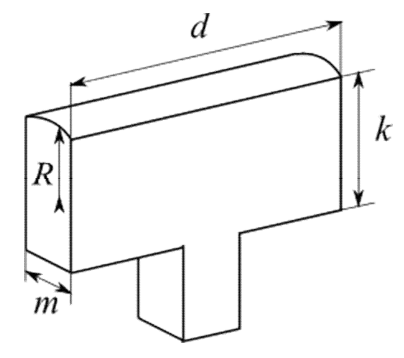

Fig. 1. Special indenter geometry.

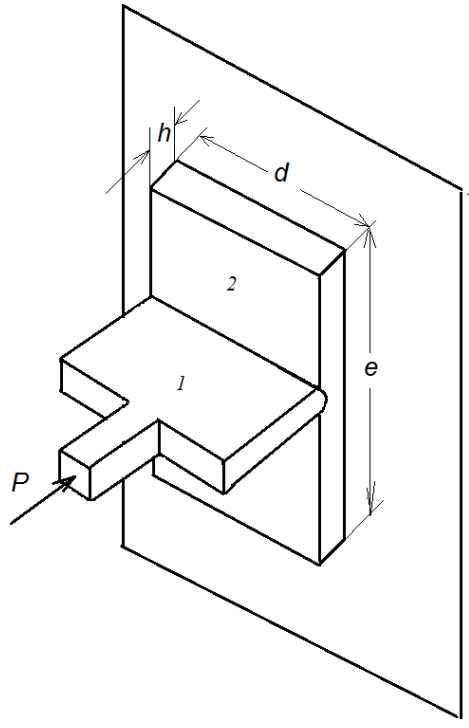

Fig. 2. Indentation scheme. 


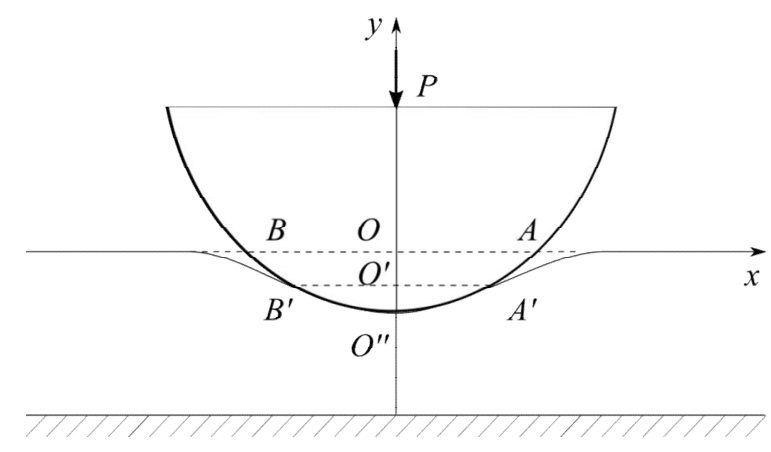

Fig. 3. Geometry of the contact problem.

The sample is a parallelepiped of length $l$, width $d$ and thickness $h$ (while $l>>d>>h$ ), which is rigidly coupled with the substrate. The width of indenter $d$ is substantially greater than the radius of curvature $R$ in the top of a parabolic (circular) cylinder. In this case, the stress-strain state arising in each cross section by the plane which is orthogonal to the generator of a parabolic (circular) cylinder represents the same behavior except for those cross sections approaching to the end of the indenter which are located on the edge of the sample. The indenter is made of steel 80 piece representing a rectangular plate. The orientation of the sample under the indenter is provided by the adjustable holder to which the sample is attached.

On the Fig. 4a, b one can see photo of the special indenter before loading (a) and under applied load (b). When the indenter is pressed into the sample, its settled ends are visible at the hardware level with video cameras with the necessary zoom and display of pictures on the screen (Fig. 5). With the help of the third video camera, the quality of the contact along the generator is controlled. Video equipment is fitted with a measurement scale, regulation of the sharpness of the image and system for required zoom of the image. Additional devices for monitoring the indentation process make possible to measure the width of the contact area $2 a$ (Fig. 4b), the depth of the contact area $\varepsilon$ (Fig. 4b) and to compare them. Other indentation parameters: indentation force $P$, indenter penetration depth $\delta$, loading velocity $v$, are set and controlled by hardware of NanoTest 600 in standard operation mode (Fig. 5). To calculate the elastic characteristics of the material sample the theoretical formulation of the problem based on mathematical modeling of the indentation process is applied using the experimental and theoretical data. 


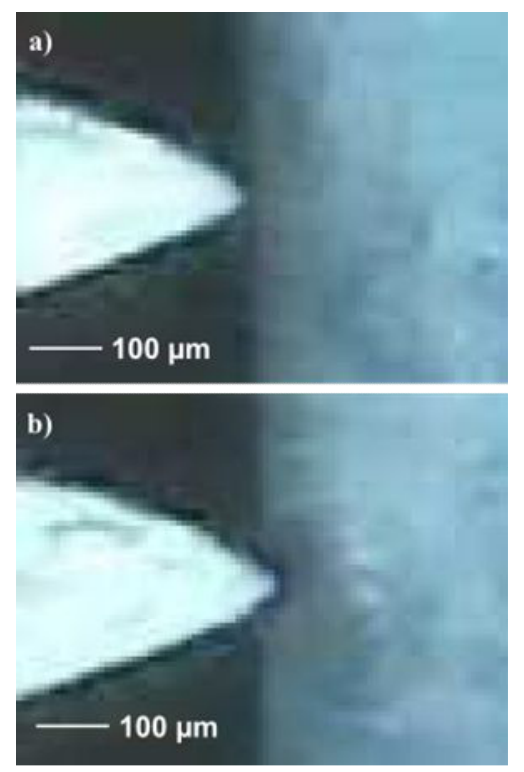

Fig. 4. Images of indentation process taken by the video monitoring system

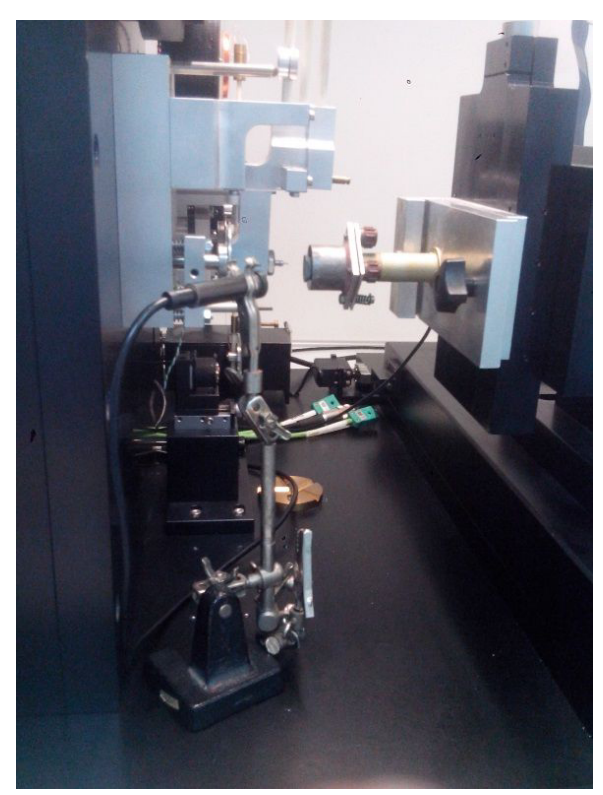

Fig. 5. Inner look of the NanoTest 600 machine

\section{Mathematical model selection}

From the description of the indentation process, the object of interest is the stress-strain state of the sample in the plane orthogonal to a generator of cylinder indenter, at distance of 2-3 times the cylinder curvature radius to indenter ends. The stress-strain state is analyzed within a plane contact problem of the theory of elasticity about pressing of a rigid indenter (punch) with parabolic (circular) base (Fig. 1) into an elastic strip (sample) with thickness $h$ bonded to a substrate.

Contact of the indenter with the strip material along its upper surface is described by a mixed boundary condition

$$
\begin{aligned}
& y=h: \quad v(x, h)=\delta-\chi x^{2} \quad|x|<a \\
& \sigma_{y y}(x, h)=0 \quad a<|x|<\infty \\
& \sigma_{x y}(x, h)=0 \quad|x|<\infty
\end{aligned}
$$

On the bottom edge of the strip with thickness $h$, which is bonded to the rigid substrate, the following conditions are fulfilled:

$$
\begin{array}{ll}
y=0 & v(x, 0)=0 \\
& u(x, 0)=0
\end{array}
$$

The solution of the problem is carried out using the integral Fourier transform [3] and it is reduced to the solution of the integral equation [4]

$$
\int_{-1}^{1} \varphi(\xi) k\left(\frac{\xi-x}{h}\right) d \xi=2 \pi \frac{\Delta}{a} f(x) \quad|x|<a, \quad \Delta=\frac{E}{2\left(1-v^{2}\right)}, \quad \lambda=\frac{h}{a}
$$

where $\varphi(x)$ are unknown stresses in the contact area, $k(t)$ is the kernel of integral equation 


$$
\begin{gathered}
k(t)=\int_{-\infty}^{\infty} K(\alpha) e^{i \alpha t} d \alpha \\
K(\alpha)=\frac{2 \kappa \operatorname{sh} 2 \alpha-4 \alpha}{\alpha\left(2 \kappa \operatorname{ch} 2 \alpha+1+\kappa^{2}+4 \alpha^{2}\right)}, \kappa=3-4 v
\end{gathered}
$$

Here in (6), (8) $E$ and $v$ are Young's modulus and Poisson's ratio of the strip material, respectively, $a=O^{\prime} A^{\prime}=O^{\prime} B^{\prime}$ is a half-width of a contact area of indenter with the strip material (Fig. 1), $f(x)=\delta-\chi a^{2} x^{2}$, where $\delta=O O^{\prime \prime}$ is the indenter immersion (Fig. 1), $\chi a^{2} x^{2}$ is a shape of indenter.

Function $K(\alpha)$ of a complex variable $\alpha=\xi+i \eta$ depends only on $v$ and is continuous by $\alpha$ on the real axis $\operatorname{Im}(\alpha)=\eta=0$, when $\alpha=0$ it has a removable singularity; when $v=0.5$ it has a double zero with $\alpha=0$; it is an even function, meromorphic in the complex plane $\alpha$ with a countable set of zeros and poles; it decreases at infinity

$$
K(\alpha)=\frac{1}{|\alpha|}+O\left(\frac{1}{\left|\alpha^{3}\right|}\right) \text { at }|\alpha| \rightarrow \infty
$$

and defined in $\alpha=0$ and its neighborhood

$$
K(\alpha)=K(0)+O\left(\alpha^{2}\right) \quad|\alpha| \rightarrow 0 \quad K(0)=\frac{1-2 v}{2\left(1-v^{2}\right)}
$$

Taking into account properties of $K(\alpha)$ and asymptotic relations (9), (10) the kernel $k(t)$ is represented as [2]

$$
\begin{gathered}
k(t)=-\ln |t|+F(t) \quad 0<|t|<\infty \\
\left.F(t)=\int_{0}^{\infty}[(1-\xi K(\xi)) \cos \xi t-\exp (-\xi)]\right\}^{-1} d \xi
\end{gathered}
$$

where $F(t+i \tau)$ is regular in the strip $|\tau|<2,|t|<\infty$, and even on $t+i \tau$.

Function $F(t)$ when $|t|<2$ is represented by an absolutely convergent series

$$
F(t)=\sum_{k=0}^{\infty} d_{k} t^{2 k}
$$

Coefficients of the series $d_{k}, k=0,1,2, \ldots$ are calculated by formulas

$$
d_{0}=\int_{0}^{\infty}\left(1-\xi K(\xi)-e^{-\xi}\right) \xi^{-1} d \xi, \quad d_{k}=\frac{(-1)^{k}}{(2 k) !} \int_{0}^{\infty}(1-\xi K(\xi)) \xi^{2 k-1} d \xi
$$

which graphs are shown in Fig. 6 depending on $v \in[0,0.5]$. 


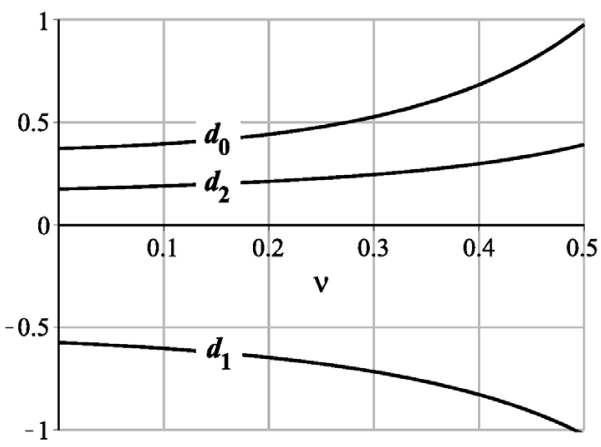

Fig. 6. Dependence of $d_{k} k=0,1,2$ on the Poisson's ratio $v \in[0,0.5]$

\section{Determination of $E$ and $v$ when $h>a$}

To determine contact stresses $\varphi(x)$ kernel $k(t)$ in form (11) is substituted into integral equation (7). Differentiation of the obtained equation with respect to $x$ reduces it to singular integral equation. After inversion of this equation one has integral equation of second kind with respect to $\varphi(x)$. The asymptotic solution of the latter [4] is built by approximation method in the form of a functional series in degrees of $\lambda=h / a$

$$
\varphi(x)=\sum_{k=0}^{\infty} \varphi_{k}(x) \lambda^{-2 k}
$$

The solution of a contact problem about penetration of a parabolic tip having $f(x)=-\delta+\chi a^{2} x^{2}$, limited at the edges of a contact area $\varphi( \pm 1)=0$, can be found from integral equation (7) in form [4]

$$
\varphi(x)=2 \Delta \chi \sqrt{1-x^{2}}\left[1-\frac{d_{1}}{\lambda^{2}}-\frac{5}{2} \frac{d_{2}-2 d_{1}^{2}}{\lambda^{4}}-\frac{2 d_{2}}{\lambda^{4}} x^{2}+O\left(\frac{1}{\lambda^{6}}\right)\right]
$$

with additional condition $\varphi( \pm 1)=0$

$$
P=\pi \Delta \chi a^{2}\left[1-\frac{d_{1}(v)}{\lambda^{2}}-\frac{3 d_{2}(v)-d_{1}^{2}(v)}{\lambda^{4}}+O\left(\frac{1}{\lambda^{6}}\right)\right]
$$

and with static condition

$$
P=a \int_{-1}^{1} \varphi(\xi)=d \xi=\pi \Delta \delta\left[\ln 2 \lambda-d_{0}+\frac{1}{2}-\frac{d_{1}}{4 \lambda^{2}}+\frac{d_{2}}{4 \lambda^{4}}+O\left(\frac{1}{\lambda^{6}}\right)\right]^{-1}
$$

Substituting $P$ from (18) into (17) one has equation to determine the Poisson's ratio $v$

$$
a^{2} \chi\left[1-\frac{d_{1}}{\lambda^{2}}-\frac{3 d_{2}-d_{1}^{2}}{\lambda^{4}}\right]\left[\ln 2 \lambda-d_{0}+\frac{1}{2}-\frac{d_{1}}{4 \lambda^{2}}+\frac{d_{2}}{4 \lambda^{4}}\right]-\delta=0, \text { where } \chi a^{2}=\varepsilon
$$

or, rewriting in more convenient notation,

$$
\begin{aligned}
& \beta\left[\theta^{4}-d_{1} \theta^{2} \beta-\left(3 d_{2}-d_{1}^{2}\right) \beta^{2}\right] \times \\
& \times\left[-\theta^{4} \ln \frac{\sqrt{\beta}}{2 \theta}+\left(\frac{1}{2}-d_{0}\right) \theta^{4}-\frac{d_{1}}{4} \theta^{2} \beta+\frac{d_{2}}{4} \beta^{2}\right]-\theta^{8}=0
\end{aligned}
$$


where $\beta=\varepsilon / \delta, \theta=h / \sqrt{2 R \delta}, d_{k}=d_{k}(v) k=0,1,2$ from (14).

On Fig. 7 there are plots of $v$ depending on $\theta, \beta$ in domain $\beta \leq(0,5 \theta)^{2}$, derived from solution of (20).

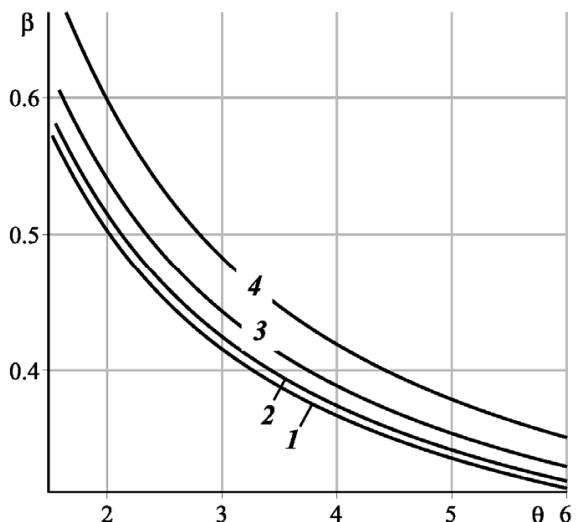

Fig. 7. Plots of Poisson's ratio $v$ depending on $\theta, \beta: 1-v=0.1,2-v=0.2$, $3-v=0.3,4-v=0.4$.

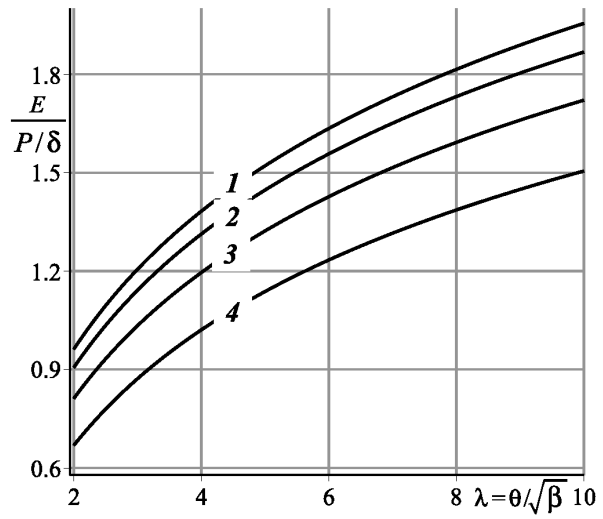

Fig. 8. Dependence of $E /(P / \delta)$ on $\lambda$ for different values of $v$ : $1-v=0.1,2-v=0.2$, $3-v=0.3,4-v=0.4$.

To determine Young modulus of the material specimen, static condition (18) is used, which gives relation

$$
E=2\left(1-v^{2}\right) \frac{P}{\pi \delta}\left[-\ln \frac{\sqrt{\beta}}{2 \theta}+\frac{1}{2}-d_{0}-\frac{d_{1}}{4} \cdot \frac{\beta}{\theta^{2}}-\frac{d_{2}}{4} \cdot \frac{\beta^{2}}{\theta^{4}}\right]
$$

To use (21), one need experimental values of $P, \varepsilon$ and $\delta$, Poisson's ratio $v$ determined from (20), and known or fixed $h$ and $R$. On Fig. 8 there are plots of $E /(P / \delta)$ derived from (21) depending on $\lambda$ for different values of $v$, which are valid in domain $\beta \leq(0,5 \theta)^{2}$.

\section{Conclusion}

The proposed method of determining the elastic parameters of the sample material, Poisson's ratio $v$ and Young's modulus $E$, can be described follows:

- during the indentation process of a specially designed indenter, the readings of standard parameters are taken, namely the load $P$, indenter offset $\delta$, speed of indenter $v$, time of indentation process $t$, and non-standard ones, which are the width of the contact area of the indenter with the material $2 a$, penetration depth of indenter $\varepsilon$, at fixed values of sample thickness $h$, radius $R$ of curvature at the top of the indenter, sample length and width $l$ and $d(l>>d)$;

- to measure non-standard parameters $a$ or $\varepsilon$ modernized nanoindenter machine NanoTest 600 Platform 3 is used with advanced optics, backlight and device for spatial orientation of specimen;

- Poisson's ratio $v$ is obtained from (20) in the most common indentation cases when $h>a$,

- Young's modulus $E$ is obtained from static condition (18) for $h>a$. 
This research was supported by grant of the Government of Russian Federation no. 14.Z50.31.0046.

\section{References}

1. W.C. Oliver, G.M. Pharr, J. Mater. Res., 7(6), 1564 (1992)

2. J.S. Field, M.V. Swain, J. Mater. Res., 8(2), 297 (1993)

3. I.N. Sneddon, Fourier Transforms (McGraw-Hill, New York, 1951)

4. I.I. Vorovich, V.M. Aleksandrov, V.A. Babeshko, Nonclassical Mixed Problems of Elasticity Theory (Nauka, Moscow, 1974, in Russian) 